László Imre Komlósi

\title{
13 Digital Literacy and the Challenges in Digital Technologies for Learning
}

\subsection{The Digital Age: A paradigm Change in Social Cognition}

The digital age has brought not only new ways of collecting and processing information for human beings (and other intelligent, reasoning entities as well) in the different societies on the globe - regardless of the social and political order or ideological commitment - but it has also made it necessary for researchers to develop new ways of thinking about and understanding digital culture in the quest for answers to complex questions about literacy and human learning and social cognition.

In the present study, I make an attemp at summarizing some of the converging findings in my research in the fields of cognitive linguistics and cognitive anthropology, which I have conducted in the course of the past decade. My interest has evolved around research on metaphorical and metonymical meaning structures together with meaning extension via conceptual integration, the theory of rationality and mental state attribution, discursive reasoning and argumentative skills development under the conditions of interactive, contextualized verbal behavior. I intend to survey briefly the reciprocal relationship between verbal behavior and social cognition in which both cognitive and affective processes play a crucial role. I plan to show how certain learning techniques get amplified, while others decrease in importance due to the changing nature of accessing and processing information in the digital era.

The paper is based on the observation that unprecedented development and innovation in information and communication technologies have exerted unforeseen impact on social cognition, information processing and human learning. We have witnessed the appearance of smart systems of various sorts whose interactive elements include reasoning (i.e., reasonably computing) entities with both human and nonhuman properties. It is realistic to claim that learning and knowledge management in digital social space are bound to take place and consist in the interaction between intelligent cognizing entities, human and non-human alike.

The paper identifies the observablecauses of a paradigm changein social cognition and infocommunication, creating a new environment for knowledge management. It is argued that the long evolutionary process of linear information processing constituting narrative-like mental structures based on cultural conceptualizations of any culturally coherent human community has already been challenged by parallel and connected network-based information processing making use of fragmented, encapsulated information chunks provided by a plethora of information sources. It is claimed that traditional learning conditions were constituted by a hierarchically determined distribution of knowledge and information and by a norm-based culture 
of behavior patterns. In opposition to the traditional learning conditions, the emerging patterns of digital cognition, digital information management and digital literacy are not only faster involving greater complexities, but they also facilitate coping with virtualization in general, leading to new cultural landscapes involving augmented reality. The new phenomenon is acknowledged in the paper by adopting the term Connected Cognitive Entity Generation (CCE Generation), by which I refer to the participants of the new information management practices induced by the very nature of the digitalized information environment. It is also claimed that the postGeneration Y is waiting in the wings, having finally broken out of the hierarchically structured, authoritative knowledge distribution due to the unprecedented advancement and development of the digital technologies. As opposed to Generation $\mathrm{X}$ or Generation Y, cognitive entities constituting the digital space as 'digital natives' will continually generate connected cognitive entities (CCEs) who will no longer be confined to generating a demographically conceived population. They will be social actors as interacting cognitive entities.

I will elaborate on the observation that cognitive entities in the digital environment are related to each other, not by commonly shared cultural narratives (as stated in the tenets of cognitive cultural anthropology to be depicted below in the following sections) but by random interest in networking, information sharing, and emergent cognition. One should also realize that this type of networked information exchange is not teleology-driven. What we witness under such conditions is an exponential growth of potential information sources due to the parallel design of connections.

After a brief survey of the different approaches to literacy, I will look at the contribution of education - formal and informal alike - and that of socialization primary and secondary - to the development and shaping of literacy. Based on the conceptual framework I have adopted and the arguments I set out to elaborate on, I will put forward a weak hypothesis and a strong hypothesis.

The weak hypothesis assumes that there is no sharp dividing line between familiarity with disciplines in the natural sciences and with disciplines in the humanities and social sciences from the point of view of information management. I presume the presence of universal mental processes in perceiving, processing and managing information of any type (be it verbal, multi-modal, concrete, abstract, figurative, symbolic, analogue or digital, etc.).

The strong hypothesis claims that there are crucial and decisive differences in how the universal mental processes are put to use with the help of flexible adaptations to context-sensitive or context-dependent parameters of mental modeling. Exploiting the vast potentials and increased efficiency of individual and social cognition provided by digital information technologies will be a landmark in learning theories. However, the development of digital competences might involve a danger at the same time by the creation of a demarcation line or socialization gap between the advantaged and the disadvantaged. The use and misuse, or the exploitation and the lack of exploitation of a new world of information flow and information processing might create the greatest 
challenge for educators and politicians in finding the right ways and pace of social integration in the near future.

\subsection{Cultural Conceptualizations and Cultural Narratives}

\subsubsection{Background assumptions}

Knowledge management has been in the focus of interest for a few decades, however, consensus among representatives of cognitive anthropology, cognitive linguistics, artificial intelligence and cognitive infocommunications culture shaped by digital communication environments has been scarce. On one hand, this can be accounted for by the different disciplinary traditions pertaining to the respective fields. On the other hand - what the theoretical orientation of our research strongly suggests - it is to be acknowledged that the difference lies in the fundamental presumptions about social cognition within the realm of cognitive anthropology and those within the realm of digital culture. My objective in this paper is to point at the crucial difference in the assumptions. It is exactly the new assumptions regarding cognition and learning in the digital culture that seem to have led to a paradigm change as an inherent property of the digital era.

For the sake of convenience and transparency, I propose a comparison between two fascinating fields of research profoundly committed to knowledge management in the social space. On one hand, I will refer to current tenets of cognitive anthropology by discussing the concept of cultural conceptualizations and situated cognition manifest in socially situated discourse (Frank, 2008) and the concept of distributed cultural conceptualizations and emergent cultural cognition (Sharifian, 2003; Sharifian, 2008; Sharifian, 2011). On the other hand, I will survey the concept of digital communication environments creating augmented virtual realities (Abrash, 2014) and the concepts of cognitive entity generation (Baranyi \& Komlósi, 2015; Baranyi, Csapó \& Sallai, 2015).

\subsubsection{Collectively constituted linear narratives with commonly shared interpretational patterns}

We have witnessed a shift in cognitively oriented studies from the individual perspective on cognition to the social and cultural embeddedness of both linguistic cognition and social cognition, which has stimulated and widened the interest of researchers to a great extent. The idea of sociocultural situatedness revealed the mechanisms of cultural conceptualization and came to be seen as the foundation of socialization and interactive communicative behavior. An outstanding tradition 
in this respect has been established by the representatives of Conceptual Metaphor Theory (Lakoff \& Johnson, 1980; Kövecses, 2005; Kövecses 2006).

The socio-cultural frame underlying the interactive communicative patterns of a community can be perceived as a mental framework providing a virtual fabric, i.e., a cohesive narrative to accommodate and determine culture-specific interpretation schemes for the members of cultural groups. Frank (2008) describes socio-cultural situatedness as a vital interaction of the individual mind with social and cultural structures (such as other agents, artifacts, conventions, etc. - in short, tangible and intangible cultural participants), thus facilitating social cognition involving learning, socialization, collective memory, mental maps of events, norms and patterns of behavior, etc. Although the nature of the interaction in the theory of socio-cultural situatedness is not spelled out in detail, one cannot fail to observe the quintessence of situated cognition: the socio-cognitive involvement of the individual mind is realised via interactions with other individual minds and with other social and cultural entities, where the community of individual minds collectively creates cohesive and supportive narratives in the course of these interactive processes.

One cannot fail to evoke at this point the foundational philosophical tradition established by Habermas's consensus-seeking communicative acts (Habermas, 1994). His starting claim is that discourse has a socially situated nature which determines and restricts communication as an interactive meaning-creating activity to be realized within the horizon of shared, unproblematic convictions, which automatically constitute consensus-generating interpretative patterns (Habermas, 1994:66). It entails - at the same time - that communicative actors are always moving within the horizon of their lifeworlds since they cannot step outside of it. Lifeworld is the invisible and indispensable background of everything we do and of everything we are.

Frank (2008) succinctly summarizes the outcome of the community of 'lifeworlds', which are the results of fairly homogeneous cultural conceptualizations: their manifestation is a culturally transmitted and linguistically organized stock of interpretative patterns. Lifeworld can be understood as a kind of non-thematic knowledge that is characterized by an unmediated certainty and a holistic constitution. It is composed of cultural patterns, legitimate social orders and personality structures, forming complex contexts of meaning.

Sharifian (2003) proposes a complex theory of cultural conceptualizations that function as collective representations to secure permanence in culture. This process, however, is dynamic and highly interactive within a cultural community as conceptualizations are represented in a distributed fashion across the minds of a cultural group. Cultural dynamics manifests itself in discourse the condition of which are bound to be permanently modified as the members negotiate and renegotiate these conceptualizations across generations. It is important to distinguish between the terms narrative and discourse. In Sharifin's theory of distributed, emergent cultural cognition (Sharifian, 2008; Sharifian, 2011), collective narratives secure permanence in culture, while discursive processes permit the renegotiation of 
cultural conceptualizations across generations. It is a novelty in the formulation that renegotiated and augmented cultural narratives reveal emergent properties in the flow of cultural interactions. Sharifian's dialectically integrative view sees cognition as a complex adaptive system that emerges from the interactions between the actors of a cultural group who negotiate and renegotiate their emergent cultural cognition across time and space. Although this theory offers an interesting perspective on augmented cultural narratives that overarch generations, it does not explain what exactly guarantees permanence in collective cultural representations.

It is to be acknowledged that collectively created cultural narratives have had their decisive function in shaping social cognition and social behavior across generations functioning in a linear mode of information processing. These narratives - as results of the complex adaptive cognitive systems of the members - have been successful in creating culturally controlled and augmented realities by allowing interaction and renegotiation of emergent cultural cognition. These cultural competences enabled, orientated and encouraged members of the cultural group to cope with hierarchically built, conceptually derivable (ideologically driven) meaning structures, to develop binding forces of cultural belonging and to seek permanent roles in given hierarchical structures.

The discussion that follows is meant to challenge Sharifian's attempt to support an integrative view of social cognition based on homogeneous cultural conceptualizations. The outcome of my analysis is to suggest that permanence in collective cultural representations cannot be secured under the circumstances of contemporary digital environments producing a plethora of fragmented narratives due to the alternative, parallel mode of information processing. Digital culture enables cognitive entities in the digital environment to cope with network-generated, associative meaning structures and to make sense of fragmented narratives and emergent contexts.

\subsection{Fragmented Narratives in Digital Communication}

\subsubsection{Pragmatics: reflective contextualization and context-sensitive interpretations}

There are many aspects of social interaction, however, the notion contextualization seems to be a central and unavoidable ingredient of pragmatic studies. Building contexts or observing and acknowledging contexts are results of creative mental activities. In some sense, we can assert that contexts are representations of states of affairs and situations. We can talk about the ontological foundation of representations or conceptualizations, and we can talk about their epistemological foundation as well. I proposed a systematic hierarchical relationship between foundational settings, 
ontological commitments and epistemic states in connection with contextualisation in (Komlósi, 2011; Komlósi, 2012):

1. Situations and faithful mappings of situations

2. Contextualized situations (selective mental representations of situations)

3. The linguistic context (texts and discourse depicting contextualized situations)

4. The pragmatic contexts (constructed contexts based on users' perspectives)

5. The context of social interaction and culture (social reality, knowledge of others)

6. The context of the self (figuring in individual and social cognitive situations)

7. Instantiated mental contexts (situated language use)

8. The context of the web experience (cognition in virtual reality)

I claimed that 1-2 manifest situational contexts, 3 represents a linguistic context and 4-8 realize mental contexts. Social interactive skills require and entail those higher level cognitive skills that facilitate adaptive, context-sensitive interpretations. Here, we have to observe that social cognition is a cover term for a vast number of different cognitive activities responsible for information processing and knowledge management with relative independence of the types of informational inputs. A mental context is constructed out of selected properties of mental contents, thus serving as background for evaluating the meaningfulness of particular arrangements in information structure. Linguistic structure in itself is not sufficient to determine contextual meaning. A mental context yields added value with which linguistic meaning is to be complemented.

\subsubsection{Fragmented narratives}

An array of contemporary research suggests that we are experiencing a new paradigm in perceiving, conceiving and managing information, especially with those people who have been socialized in the digital era and have internalized the very nature of digital culture. The members of the digital community function in connected networks created by a number of different types of cognitive entities. These people are cognitive entities themselves who feel at ease with digitalization and virtualisation (Abrash, 2014). In their environments, all sorts of smart devices are at their disposal such that they can cope with a plethora of information and can communicate effectively in accordance. We can assert today that these social actors function as cognitive entities. The notion cognitive entity (Baranyi \& Komlósi, 2015; Baranyi, Csapó \& Sallai, 2015) encompasses both human and non-human agents, digital and mechanical entities that behave smartly and who/which are presumed to exist within a highly interlocked framework of a multi-channel information flow and exchange.

Cognitive entities in a digital environment are related to each other not by commonly shared cultural narratives (as stated in the tenets of cognitive cultural anthropology earlier), but by random and spontaneous interest in networking, 
information sharing and emergent cognition. We may say that this type of networked information exchange is not teleology-driven. What we witness under such conditions is an exponential growth of potential information sources due to the parallel design of connections.

To continue the metaphorical description of 'collectively constituted cultural narratives', in the case of the community of cognitive entities, we can talk about chunks of information that might be seen as fragmented narratives. Any piece of information can be related to any other information by creating suitable or agreeable contexts for interpretation (see the description of mental context above). Therefore, we can still envisage information chunks that might fit into narratives of some sort. However, the narratives created online are not based on negotiation, let alone renegotiation. They are results of non-deterministic interpretations accepted for current states of affairs. These information chunks might be ephemeral and seemingly arbitrary. Creating contexts for interpretation means an innovative challenge for the interactive agents. Fragmented narratives become flexible building blocks for novel contexts. As opposed to cultural narratives, which are based on cultural conceptualizations and are linear in design, fragmented narratives are non-linear, non-deterministic and distributed in a parallel design.

It is important to observe that the social perspective of cognition adopts the view that human cognitive abilities, especially human intelligence, are emergent properties. Such properties originate both from embodiment (i.e., mental attributes are inseparable from the physical, biological, neurophysiological bases of human existence) and from the interaction between humans, devices, artifacts, infrastructure and environment. Understanding the nature of co-evolving and emergent cognitive capabilities will legitimate the concept of cognitive entity to be developed further in the near future.

\subsection{Emergent Properties in Learning and Knowledge Acquisition}

This study is to show that the effect of digitization on the social network of connected cognitive entities holds many implications. Social cognition is to be understood as a permanent learning process, which requires higher-level cognitive skills to process and integrate emergent properties arising in the digital space through the interaction of connected cognitive entities. I want to emphasize the innovative drive which resides in the interactive informational frameworks, which consist of a multitude of connections of a cognitive personality to other cognitive entities, human and nonhuman alike. 


\subsection{Digital Literacy and The Digital Gap - Challenges for Traditio- nal Socialization Patterns}

As we have seen from the analysis in the previous section, networked digital communication environments create novel conditions for learning and knowledge management. The cognitively determined personalities in an emerging network of smart cognitive entities facilitate non-linear, multidirectional, horizontal communicative interaction among the social actors and substantially weaken the vertical, authoritative dependency lines in communication and social behavior.

It has become widely accepted that we talk about the emergence of a new generation of cognitive entities or generation CE in analogy with the generations $\mathrm{X}$, $\mathrm{Y}$ and $\mathrm{Z}$. The members of generation $\mathrm{CE}$ are different if not unique in the sense that both their primary and secondary socialisations take place in an environment that is inseparable from cognitive infocommunications in the digital space.

As a result of my investigation, I am claiming that situated discourse based on cultural conceptualizations functioning as a uniquely determining aspect of social communicative acts has lost its explanatory power due to the heterogeneous character of the information sources in the digital environment. No exclusively determining cultural conceptualizations exist anymore, which would function as the ontological basis for shared convictions.

However, I have a piece of good news to share, too. I can claim that connected cognitive entities do share a common ground for communication, which draws on the emergent properties of infocommunications, i.e., the thrill of being continually connected to and being engaged in a throbbing digital environment.

We need to realize that the long evolutionary process of linear information processing resulting in and constituting narrative mental structures based on cultural conceptualizations of any culturally coherent human community has already been challenged by parallel and connected network-based information processing making use of fragmented, encapsulated information chunks provided by a plethora of information sources.

Creating contexts for interpretation means an innovative challenge for the interactive agents. Fragmented narratives become flexible building blocks for novel contexts. Whereas cultural narratives based on cultural conceptualizations are linear in design, fragmented narratives are non-linear, non-deterministic, and are distributed in a parallel fashion by design.

All these promising aspects may unfold if the respective societies provide for supportive social environments and appropriate infrastructure for all. Here, we have to be on our guards and see that the widely experienced socio-economic gap should not be accompanied by a growing digital gap. In this paper too, I intend to call attention to the undesirable phenomenon of the digital gap in education closely connected to the phenomenon of marginalization in terms of socio-economic status. 
The role and responsibility of the educational system has to grow to avoid the negative consequences of exclusion and marginalization of certain social groups in society.

\subsection{Conclusions}

A report on contemporary research on the nature and consequences of digital culture seems to be timely as the scientific communities want to know more about the unprecedented development and innovation in information and communication technologies, which have already exerted unforeseen impact on social cognition, information processing and human learning. Due to the appearance of smart systems of various sorts whose interactive elements include reasoning entities with both human and non-human properties, it is to be expected that new ways of learning and knowledge management will be required soon.

The paper attempts to identify the causes of a paradigm change in social cognition and infocommunications, which creates a new environment for knowledge management. It is argued that the long evolutionary process of linear information processing constituting narrative-like mental structures based on cultural conceptualizations of any culturally coherent human community has already been challenged by parallel and connected network-based information processing making use of fragmented, encapsulated information chunks provided by a plethora of information sources.

The paper offers a comparison between traditional learning conditions, which were constituted by a hierarchically determined distribution of knowledge and information and a norm-based culture of behavior patterns and the emerging patterns of digital cognition, digital information management and digital literacy. The study reports on the new phenomenon called Connected Cognitive Entity Generation (CCE Generation).

The main argumentative thread of our research is that we see digital culture as a novel environment for social cognition that inevitably undermines the permanence of cultural narratives. Cognitive entities seek connection to each other in a parallel, but non-deterministic fashion in order to exploit the immense potential in a plethora of information sources. The consequences for the nature and mechanisms for social cognition based on digital culture cannot be predicted in a reliable way as of today. A new perspective on and an unprecedented practice of information management is, however, a part of an irreversible process.

The paper identifies the characteristics of the post-Generation $\mathrm{Y}$ in relation to information processing and knowledge management in order to support the growing awareness of the digital age and the assumption that socialization and acculturation in the digital era rests on a significant paradigm change in the realm of social cognition. Digital culture is claimed to be acquired by the members of modern societies in a smooth and seamless way, the result of which is the permeability between human 
agents and non-human agents in the contexts of information processing and communicative interaction. The paper calls attention to the undesirable phenomenon of the 'digital gap in education and socio-economic status' which is a consequence of exclusion and marginalization of certain social groups in society.

\section{References}

Abrash, M. (2014) “What VR could, would, and certainly will be within two years," USA: Valve Corporation / http://blogs.valvesoftware. com/abrash/. Retrieved on 26.09.2015

Baranyi, P. and A. Csapo (2012): Definition and Synergies of Cognitive Infocommunications, Acta Polytechnica Hungarica Vol. 9/ 1, pp. 67-83

Baranyi, P. and L. I. Komlósi (2015): “Connected Cognitive Entity and the CE Generation: Emergent properties in the digital space," (forthcoming)

Baranyi, P., A. Csapo and G. Sallai (2015): Cognitive Infocommunications (CogInfoCom). Berlin: Springer International Publishing.

Frank, R, M. (2008): “Introduction: Sociocultural Situatedness,” in: Body, Language and Mind. Sociocultural Situatedness, Vol. II, R. M. Frank, R. Dirven, T. Ziemke, E. Bernárdez, eds., Berlin/ New York: Mouton de Gruyter, pp. 1-18.

Habermas, J. (1994): “Actions, speech acts, linguistically mediated interactions and the lifeworld”, in: Guttorm Floistad (ed.) Philosophical Problems Today, Vol. 1. Dordrecht/Boston: Kluwer, pp. 45-74.

Komlósi, L.I. (2011): Contextualization and Cognitive Synergism. The Interaction of Ontology and Epistemology in the Interpretation of Contexts. In: Hölker, K. und C. Marello (Hrsg): Dimensionen der Analyse von Texten und Diskursen/Dimensioni dell'analisi di testi e discorsi. Festschrift für János Sándor Petöfi zum achtzigsten Geburtstag/Festschrift per János Sándor Petöfi in occasione del suo ottantesimo compleanno. Berlin/London/Zürich/Wien: LIT Verlag, pp. 186-203.

Komlósi, L.I. (2012) Linguistic Context, Pragmatic Context, Mental Context: Meaning Construction and Interpretation via Contextualization. In: Dontcheva-Navratilova, Olga and Renata Povolná (eds): Discourse Interpretation: Approaches and Applications. Newcastle upon Tyne: Cambridge Scholars Publishing, pp. 19-38.

Kövecses, Z. (2005): Metaphor in Culture. Universality and Variation. Cambridge: Cambridge University Press.

Kövecses, Z. (2006): Language, Mind, and Culture. A Practical Introduction. Oxford: Oxford University Press.

Lakoff, G. and Johnson, M. (1980): Metaphors we Live by. Chicago: The University of Chicago Press.

Sharifian, F. (2003): “On cultural conceptualizations," in: Journal of Cognition and Culture, Vol. 3.3. Leiden: Koninklijke Brill NV, 2003, pp. 187-207.

Sharifian, F. (2008): “Distributed, emergent cultural cognition, conceptaulisation and language,” in: Body, Language and Mind. Sociocultural Situatedness, Vol. II, R. M. Frank, R. Dirven, T. Ziemke, E. Bernárdez, eds., Berlin/New York: Mouton de Gruyter, pp. 241-268.

Sharifian, F. (2011): Cultural Conceptualization and Language. Amsterdam: John Benjamins. 